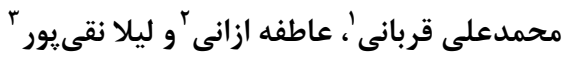

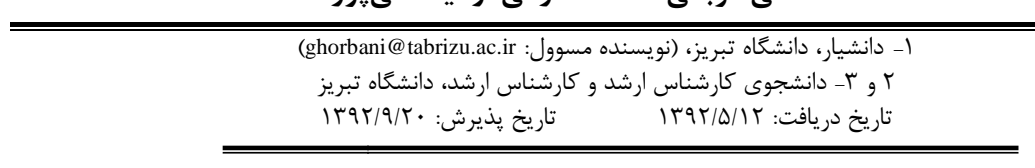

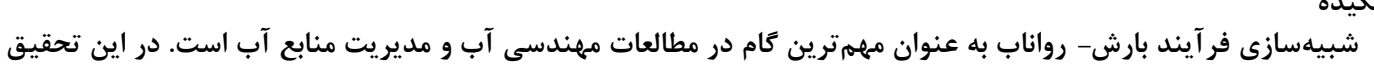

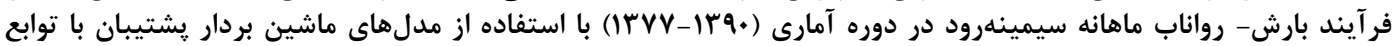

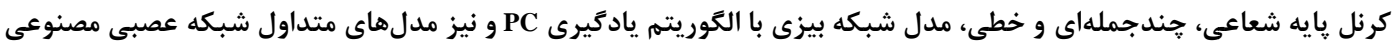

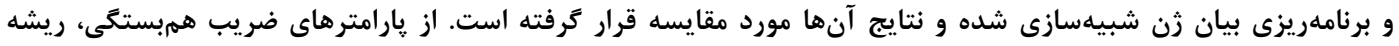

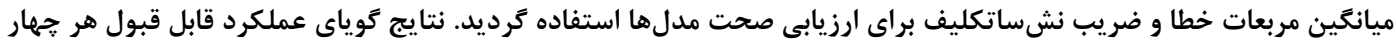

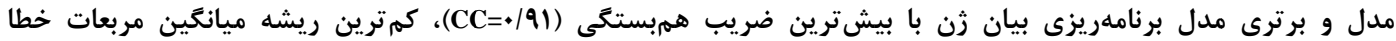
(RMSE=r// m³/s)

وازههاى كليدى: بارش- رواناب، برنامهر يزى بيان زن، سيمينهرود، شبكه بيزى، شبكه عصبى مصنوعى، ماشين بردار پشتيبان

بيان زن در شبيهسازى فرآيند بارش- رواناب روزانه

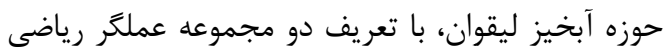

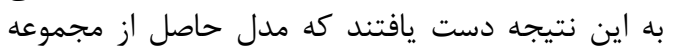

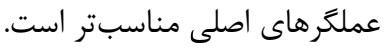

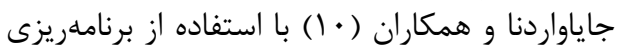

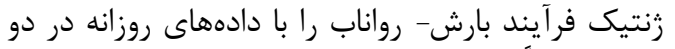

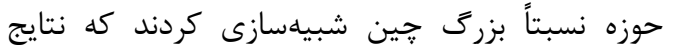

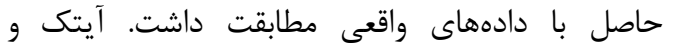

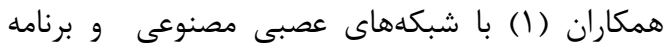

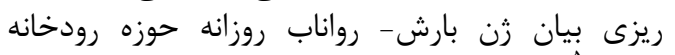

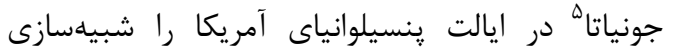

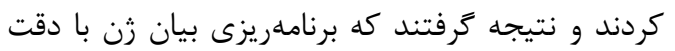

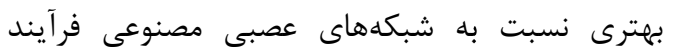

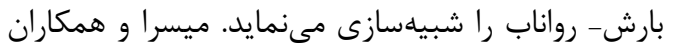

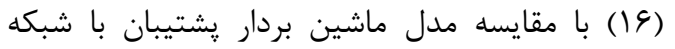

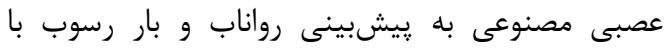

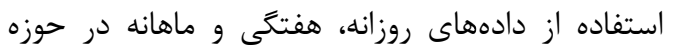
آبخيز هند برداختند.

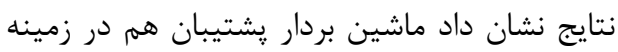

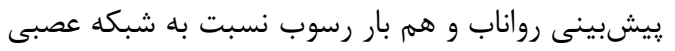

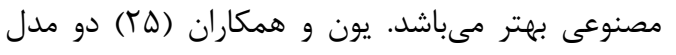

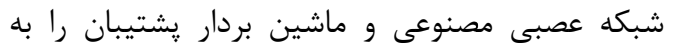

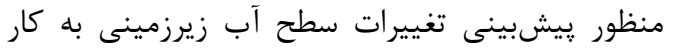

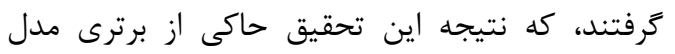

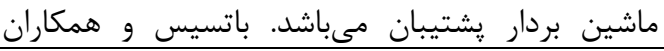
1- Artificial Neural Network 4-Support Vector Machine

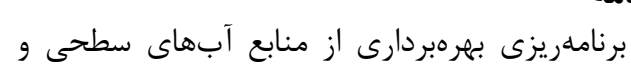

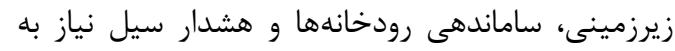

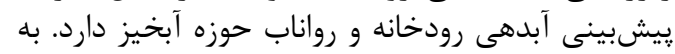

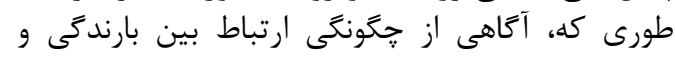

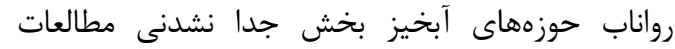

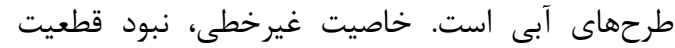

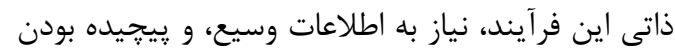

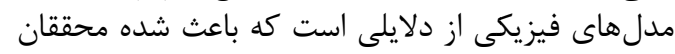

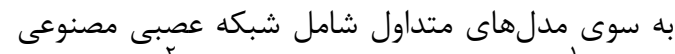
(ANN)

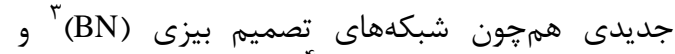

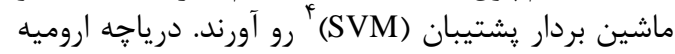

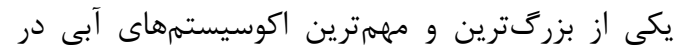

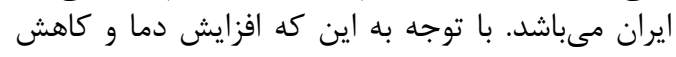

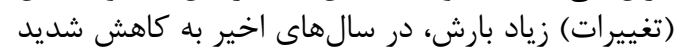

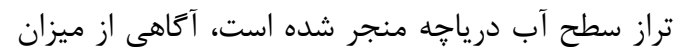

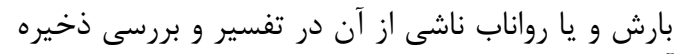

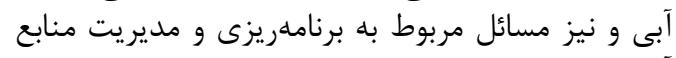

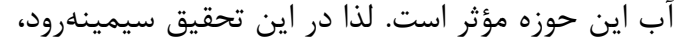

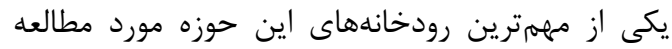

قرار كرفته است.

مطالعات متعددى با روشهاى ذكر ذر شدر شدر در زمينه

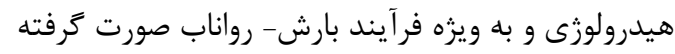

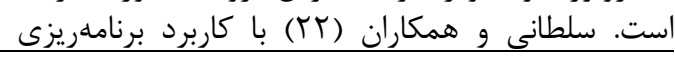
2- Gene Expression Programming 5- Juniata 
به يك جواب بهينه كلى منجر ميى مردد. در مدل ركرسيون SVM تابعى مرتبط با متغير وابسته

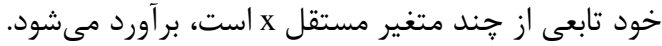

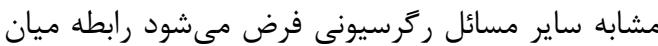

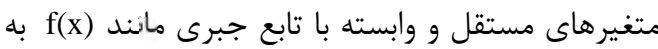

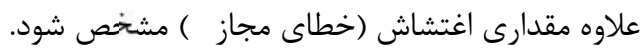
$\mathrm{f}(\mathrm{x})=\mathrm{W}^{\mathrm{T}} \cdot \emptyset(\mathrm{x})+\mathrm{b}$

(1) (1) (1) $\mathrm{y}=\mathrm{f}(\mathrm{x})+$ noise

(ب) رابطه (T)

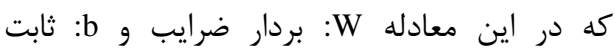
مشخصههاى تابع ركرسيونى و Øا: نيز تابع كرنل باشدا

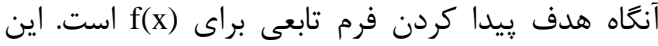

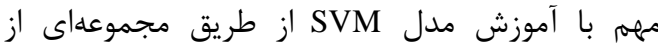
نمونهها (مجموعه آموزش) محقق مد معىشود. براى محاسبه

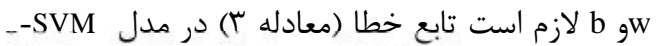

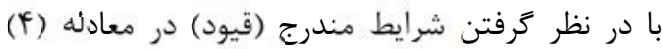
بهينه شود. $W^{T} . \emptyset\left(X_{i}\right)+b-y_{i} \leq \varepsilon+\varepsilon_{i}^{*}$ رابطه (r) (i) رابطه (F)

$\frac{1}{2} W^{T} \cdot \mathrm{W}+\mathrm{C} \sum_{i=1}^{N} \varepsilon_{i}+$

${ }^{2} \sum_{i=1}^{N} \varepsilon_{i}^{*} y_{i}-W^{T} \cdot \emptyset\left(X_{i}\right)-b \leq \varepsilon+$ $\varepsilon_{i} \varepsilon_{i}, \varepsilon_{i}^{*} \geq 0, i=$

$1,2, \ldots, N$

در معادلات بالا C C عددى صحيح و مثبت است، كه

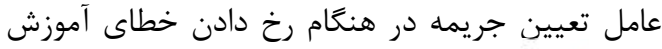

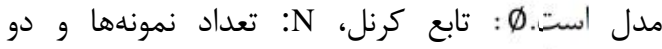

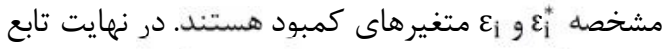
ركرسيونى را مى توان به فرم زير بازنويسى كرد: SVM

$\mathrm{f}(\mathrm{x})=\sum_{\mathrm{i}=1}^{\mathrm{N}} \bar{\alpha}_{\mathrm{i}} \emptyset\left(\mathrm{x}_{\mathrm{i}}\right)^{\mathrm{T}} . \varnothing(\mathrm{x})+\mathrm{b} \quad$ (D)

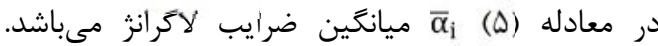

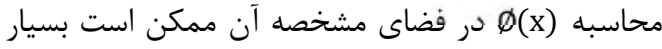

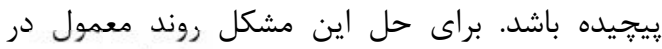

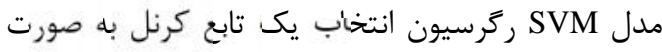

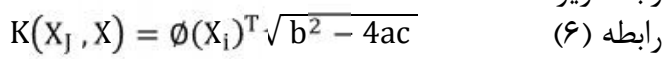
مىتوان از توابع مختلف كرنل براى ساخت انواع

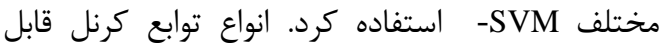

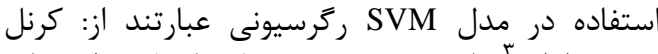

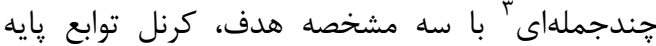

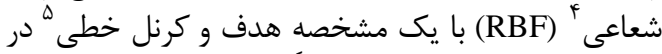

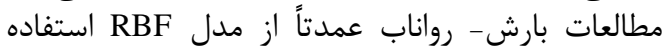

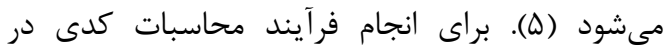
محيط متلب نوشته شده است.

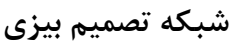

شبكه بيزى يك الكوى نمودارى احتمالاتى است كه تهن

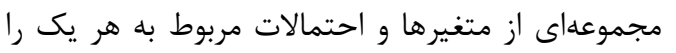

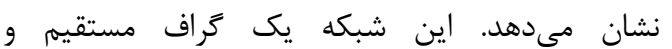

1- Standard Precipitation Index

4- Radial Basis Function

2- Active Learning Method

5- Linear
بارش- رواناب روزانه در كاليفرنيا را شبيهسازى كردند،

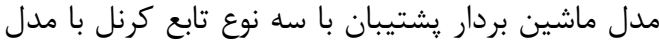

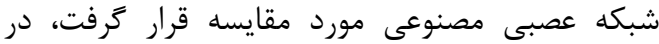

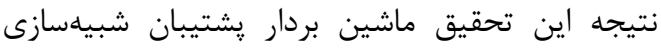

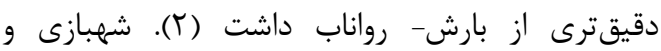

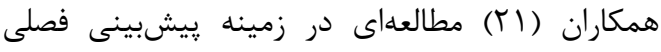

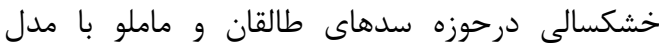

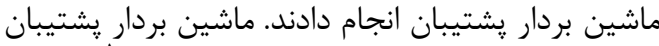

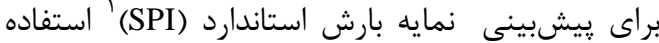

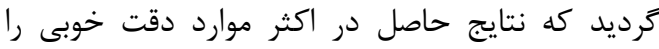

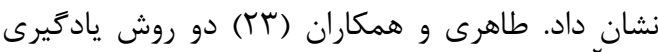

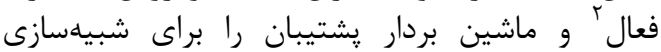

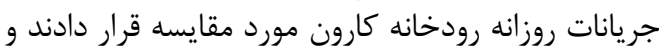

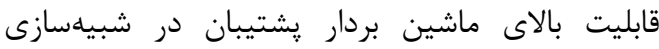
رواناب را مطرح نمودند. مهاجرانى و همكاران (IV) باردئيسان

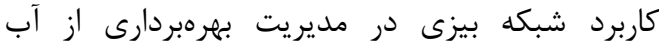

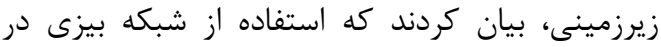

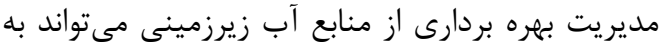

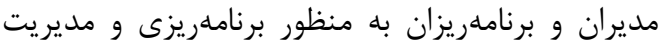

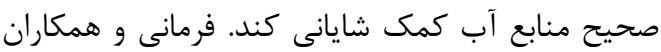

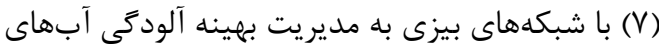

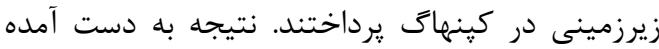

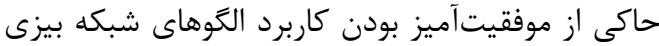

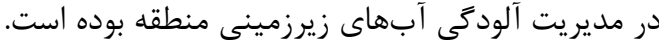

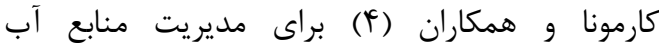

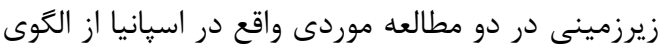

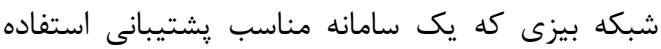

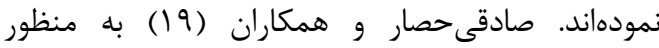

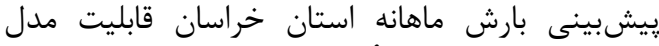
شبكه بيزى را مطرح و تأئيد نمودهاند. بـاندان

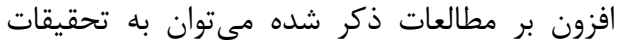

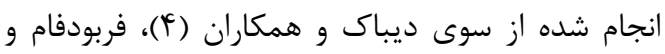

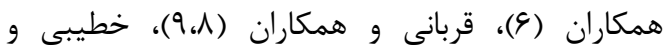

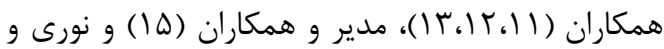

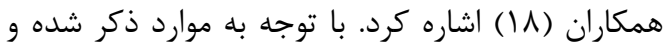

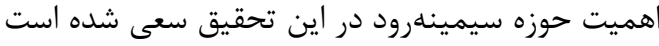

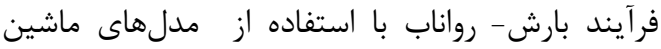

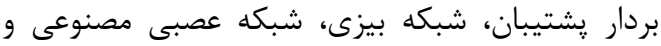

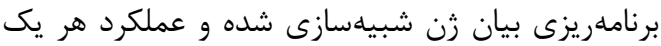
مورد بررسى قرار خيرد.

مواد و روشها مدل ماشين بردار يشتيبان

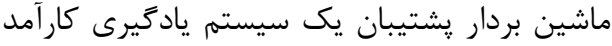

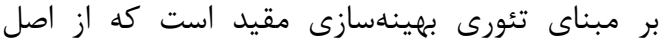
استقراى كمينهسازى خطاى ساختارى استفاده كرده و 3- Polynomial 
كه در آن، (a)

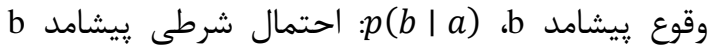

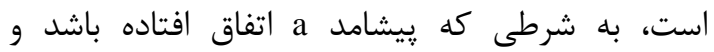

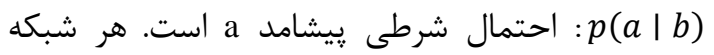

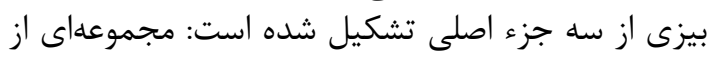

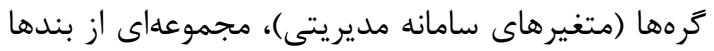

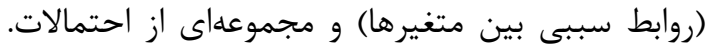

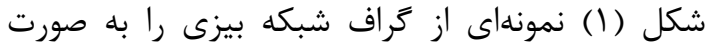
جرخهاى و مستقيم نشان مى دهد.
غير جرخهاى است كه در آن گرهها در حكم متغيرهاى

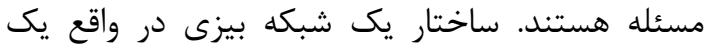

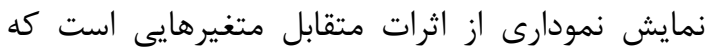

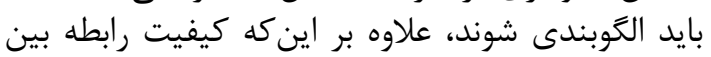

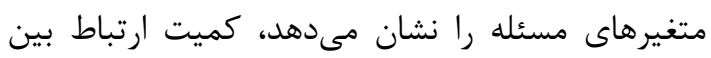

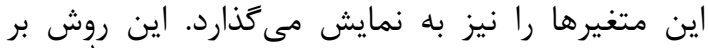

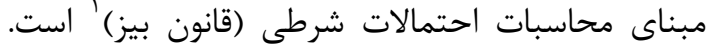

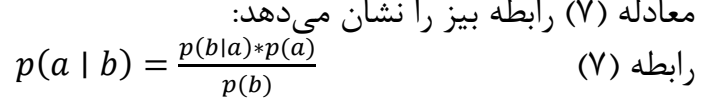

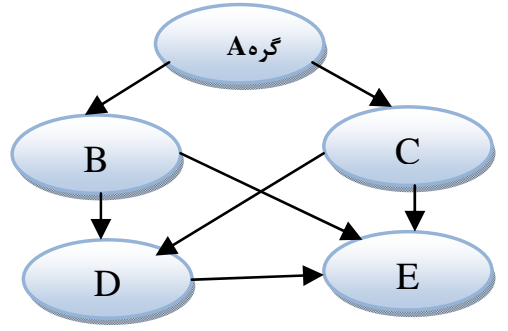

شكل 1- نمايش كراف خرخهاى و مستقيم شبكه بيزى.

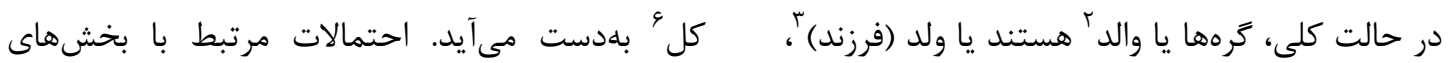

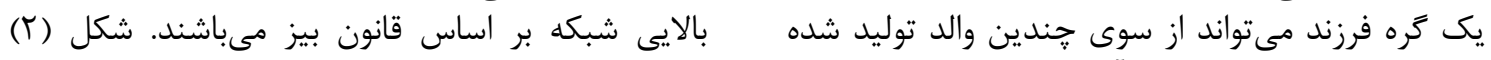

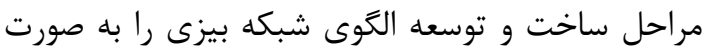

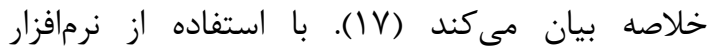
تساختار شبكه بيزى تشكيل و نتايج اين

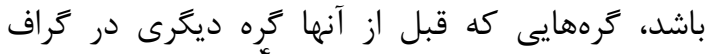

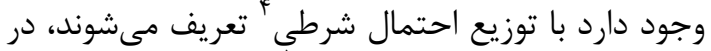

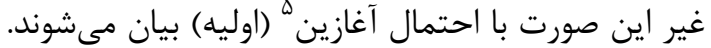
تحقيق حاصل شده است.

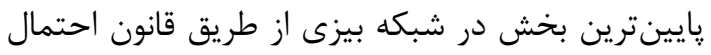

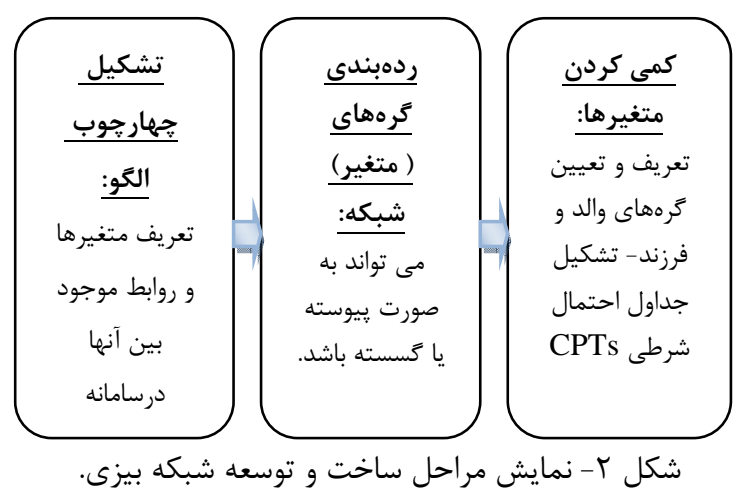

ثابت تصادفى)، Y- مجموعه عملكرهاى رياضى مورد

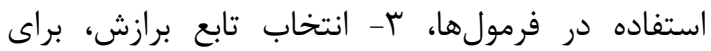

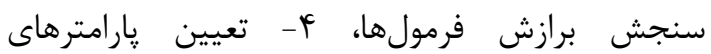

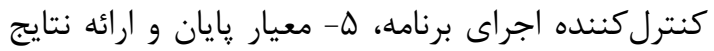

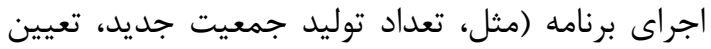

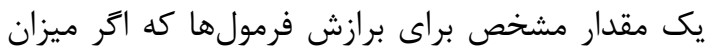

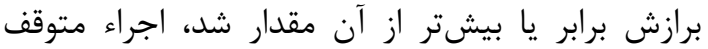

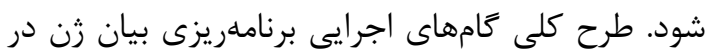

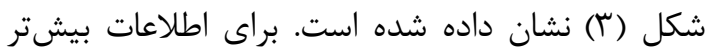
در اين مورد مى توان به راده شفره است (Yr) مراي اطعه نمود.

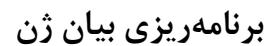

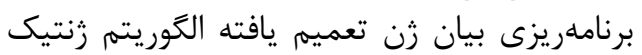

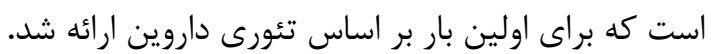

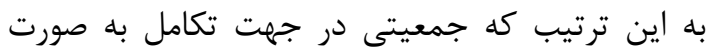

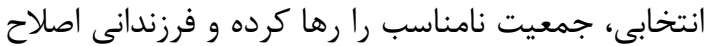

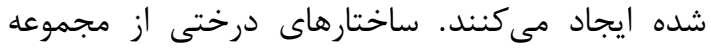

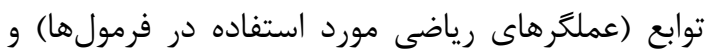

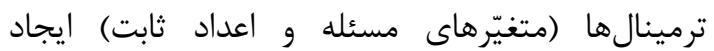

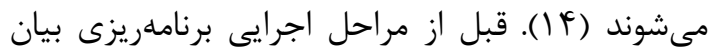

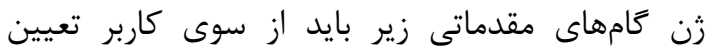
شوند: ا- مجموعه ترمينالها (متغيّرهاى مسئله، اعداد 


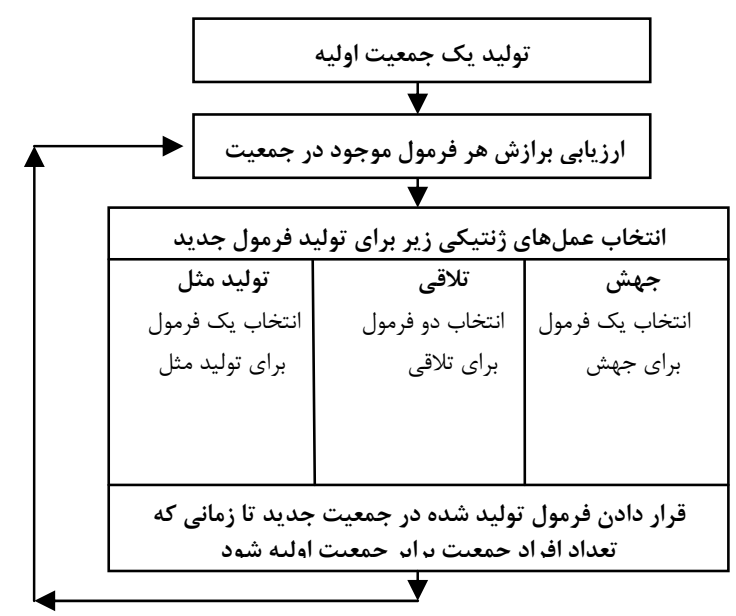

شكل r- مرورى بر شكل كلى گامهاى اجرايى مدل برنامهريزى بيان زن (Tr).

شبكه مىباشد، لايه خروجى محل استقرار يارامترهاى

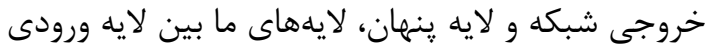

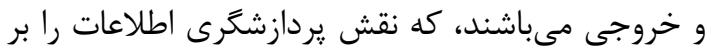
عهده دارد.
در اين تحقيق در راستاى شبيلهسازى فرآيند

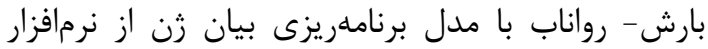
GeneXproTools 4.0 شبكه هاى عصبى مصنوعى

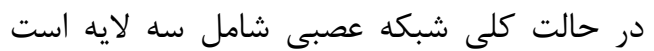

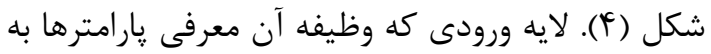

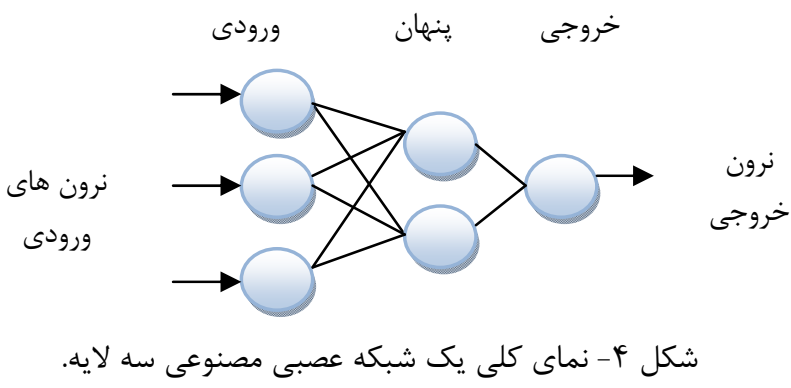

پارامترها در شبكه يرسيترون جند لايه از طريق سيخنال

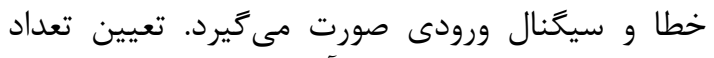

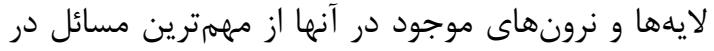

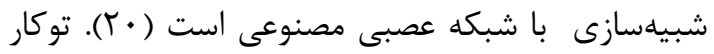

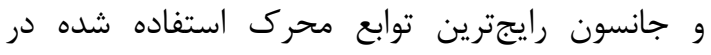

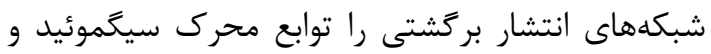
تانزانت هييربوليك ذكر كردند (YF) معيارهاى ارزيابى تئى

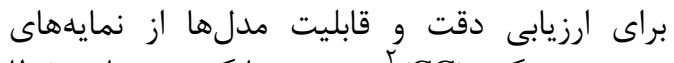

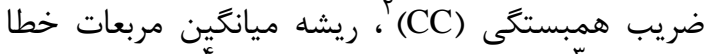
(RMSE) و و · اصورت گرفت. بهترين مقدار براى اين سه معيار

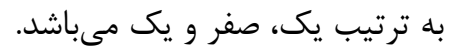

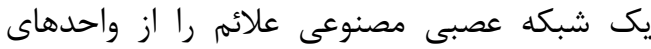

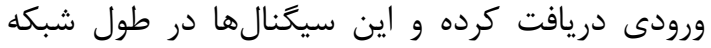

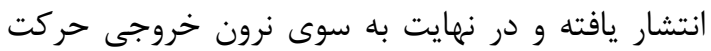

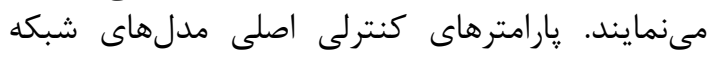

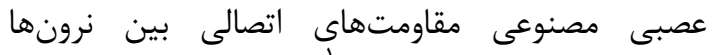

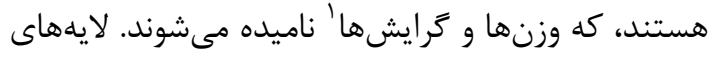

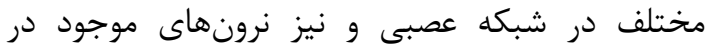

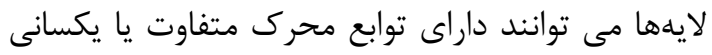
باشند. شبكههاى عصبى از نظر ساختار و ارتباطات بين مجرين

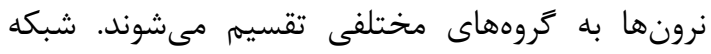

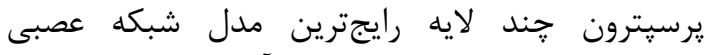

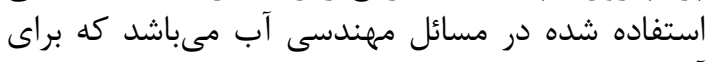

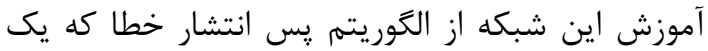

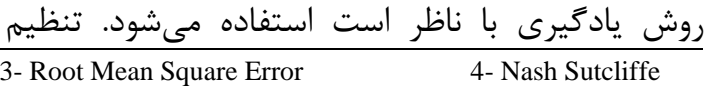


معرفى منطقه و دادههاى مورد استفاده

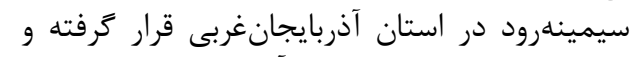

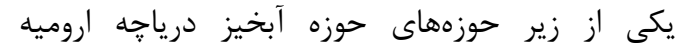

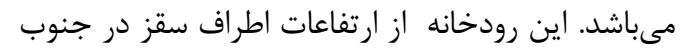

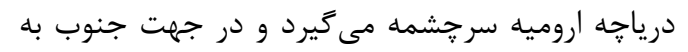

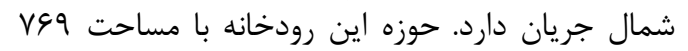

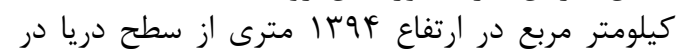

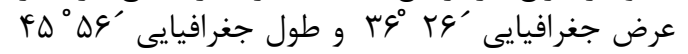

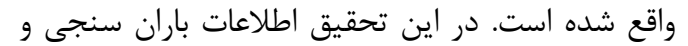

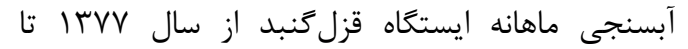

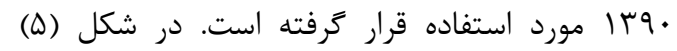

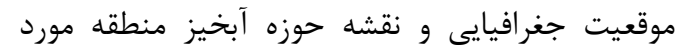

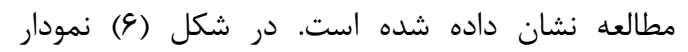

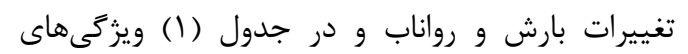

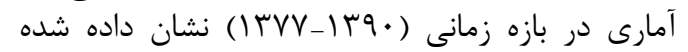

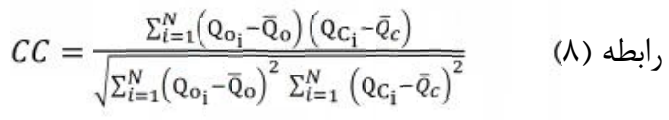$$
\text { RMSE }=\sqrt{\frac{1}{N} \sum_{i=1}^{N}\left(\mathrm{Q}_{\mathrm{o}_{\mathrm{i}}}-\mathrm{Q}_{\mathrm{C}_{\mathrm{i}}}\right)^{2}} \quad \text { (9) }
$$$$
N S=1-\frac{\sum_{i=1}^{N}\left(\mathrm{Q}_{o_{\mathrm{i}}}-\mathrm{Q}_{\mathrm{C}_{\mathrm{i}}}\right)^{2}}{\sum_{i=1}^{N}\left(\mathrm{Q}_{\mathrm{o}_{\mathrm{i}}}-\overline{\mathrm{Q}}_{c}\right)^{2}} \quad(1 \cdot) \text { رابطه }
$$

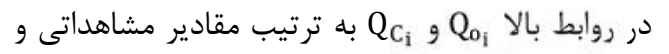
محاسباتى رواناب ماهانه در كام زمانى أiام، دادهها، ماسباتى و و $\bar{Q}$

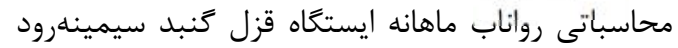

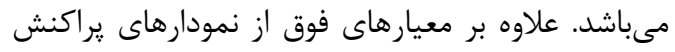

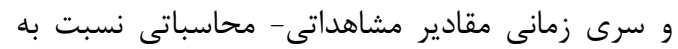
زمان جهت مقايسه و تحليل بيشتر استفاده شده است.
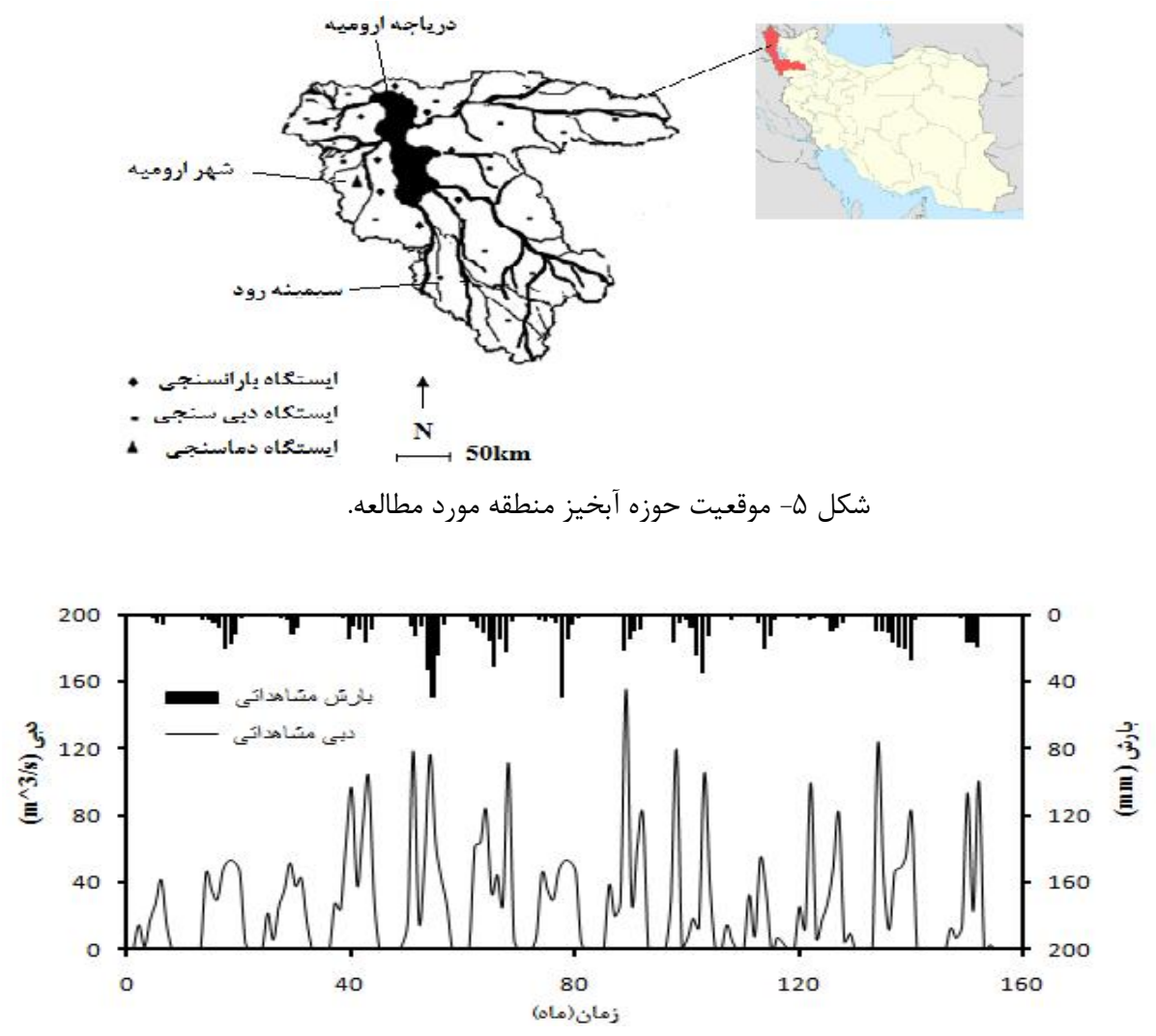

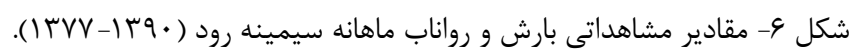




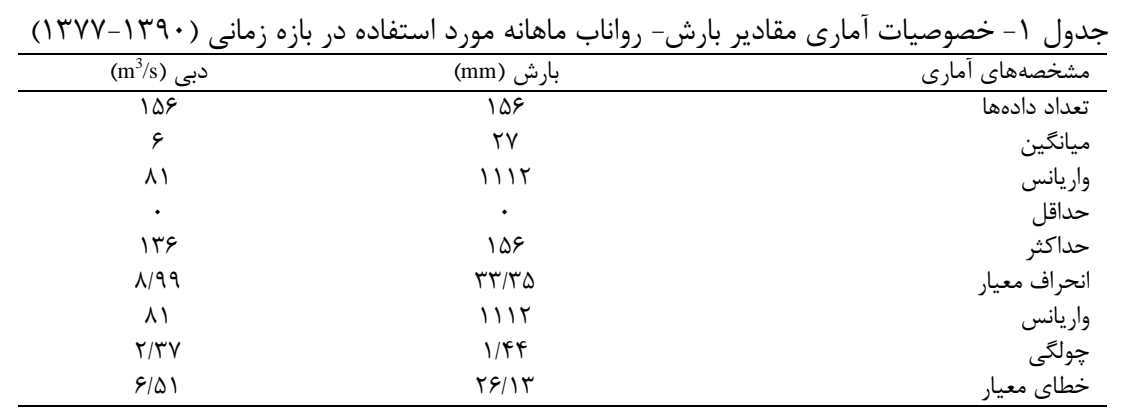

يايه شعاعى - كه از انواع رايج مورد استفاده در

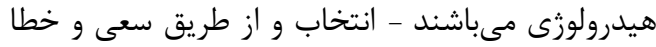

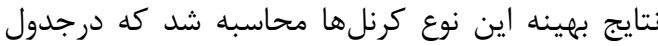

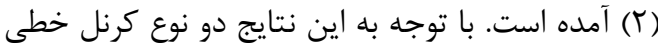

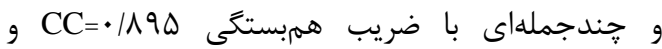

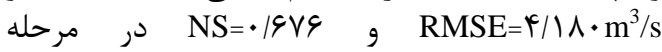
صحتسنجى عملكرد تقريباً يكسانى داشته و تابع ڤايه

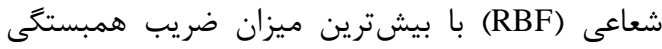

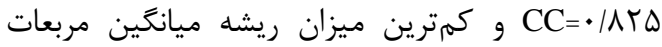
خطا

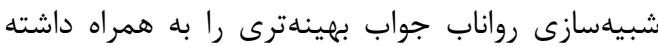

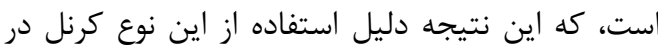

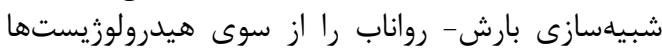

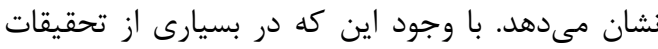

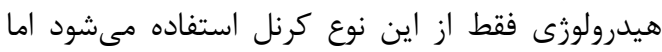

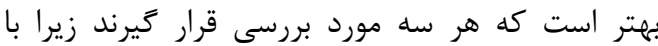

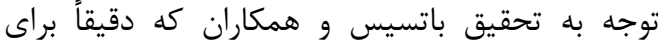

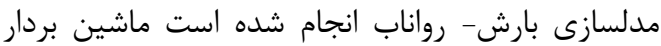

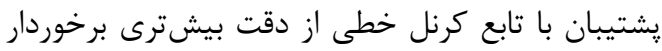

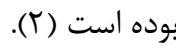

نتايج و بحث در اين تحقيق به منظور بروسى و و مقايسه نتايج

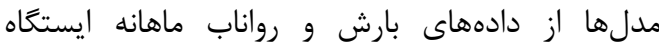

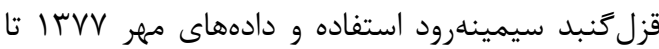

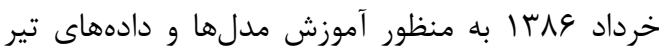

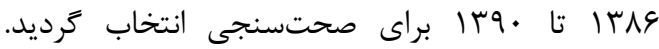

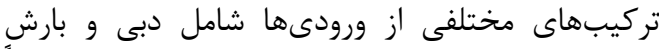

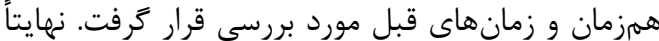

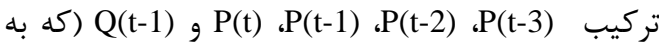

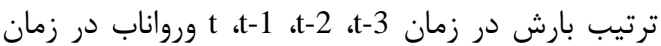

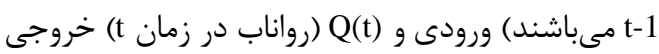

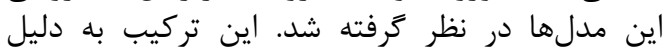

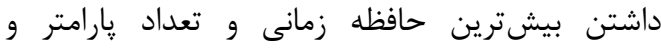

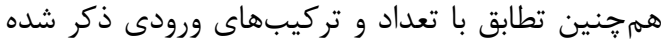

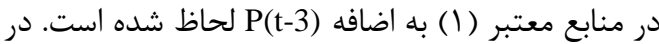
ادامه نتايج حاصل از مدل مداى مورد استفاده ارائه مي -

كردد.

مدل ماشين بردار يشتيبان

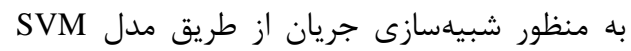

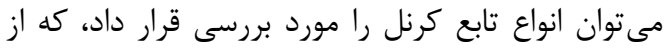

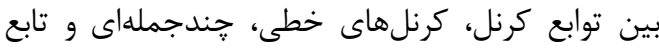

\begin{tabular}{|c|c|c|c|c|c|c|}
\hline \multicolumn{3}{|c|}{ صحتسنجى } & \multicolumn{3}{|c|}{ آموزش } & \multirow[b]{2}{*}{ كرنل } \\
\hline $\mathrm{CC}$ & $\underset{\left(\mathrm{m}^{3} / \mathrm{s}\right)}{\mathrm{RMSE}}$ & NS & $\mathrm{CC}$ & $\underset{\left(\mathrm{m}^{3} / \mathrm{s}\right)}{\mathrm{RMSE}}$ & NS & \\
\hline - IATY & $\varphi / \backslash \Lambda$. &. $\mid 9 \vee 9$ & $\cdot / V<q$ & G/TQF &.$/ D F G$ & קند جملهاى \\
\hline - MATT & $F /|\Lambda|$ & $.19 \vee 4$ & - $/ V \notin q$ & $\varepsilon / r \Delta \Delta$ & - $/ \Delta+4$ & خطى \\
\hline - IATD & $f / 1 \Delta q$ & $.19 \vee 9$ & $\cdot|V| \mid$ & $g / T \Delta F$ &.$/ D F 4$ & تابع پايه شعاعى \\
\hline
\end{tabular}

يكديخر، به صورت شكل (V) تشكيل شد و جهت فلشها

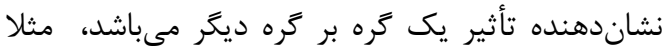

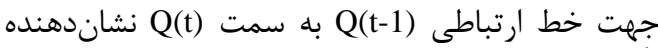

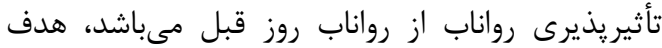

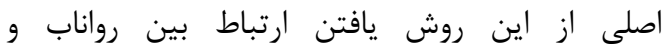

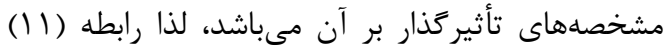

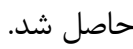

شبكه بيزى از مزيتهاى عمده اين روش مدل بـازىى، مىتوان به إنها

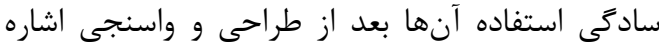

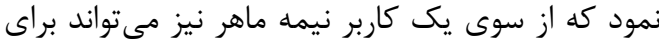

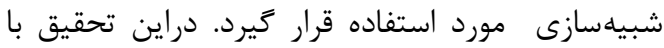

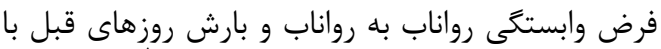

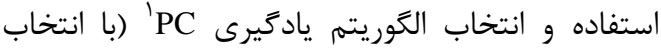

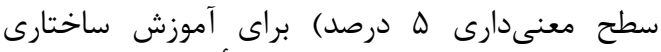

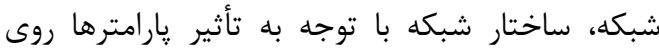


$\mathrm{Q}(\mathrm{t})=0.0169268 * \mathrm{P}(\mathrm{t}-3)-0.0181073 * \mathrm{P}(\mathrm{t}-2)+0.0365877 * \mathrm{P}(\mathrm{t}-1)+0.139917 * \mathrm{P}(\mathrm{t})+0.36635 * \mathrm{Q}(\mathrm{t}-1)+\operatorname{Normal}(-1.14677,6.35126)$

) رابطه (1)

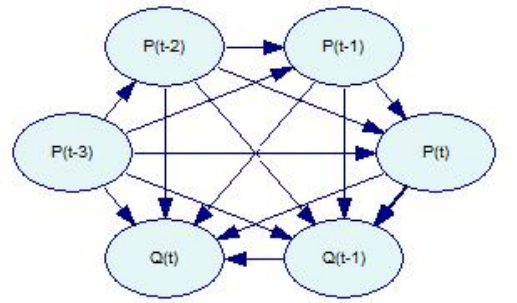

$$
\text { شكل V- ساختار شبكه بيزى به كار رفته براى شبيهسازى بارش- رواناب. }
$$

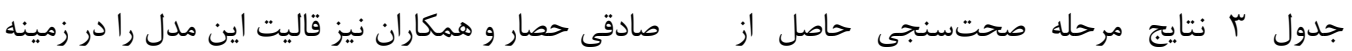

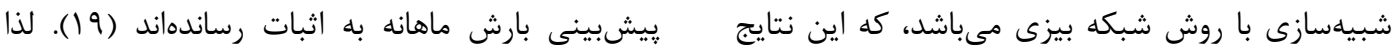

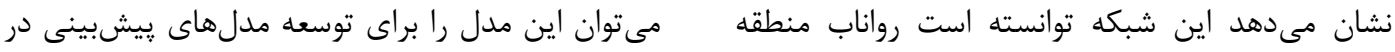

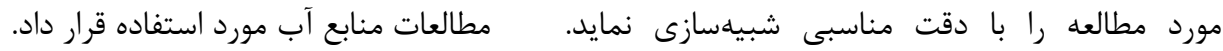

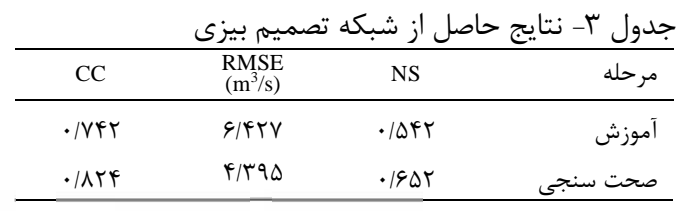

$\mathrm{F}_{1}:\left\{+,-, *, /, \sqrt{ }, \operatorname{Exp}, \operatorname{Ln}^{2}, 3, \sqrt[3]{,} \operatorname{Sin}, \operatorname{Cos}, \operatorname{Atan}\right\}$ $\mathrm{F}_{2}:\{+,-, *, /\}$

$\mathrm{F}_{3}:\left\{+,-, *, /, x^{2}\right\}$

$\mathrm{F}_{4}:\left\{+,-, *, /, x^{3}\right\}$

يارامترهاى مورد استفاده و نرخ آنها در استخراج

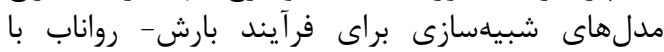

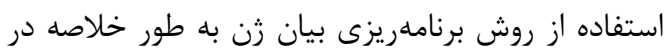

جدول (أ) ارائه شده است.
برنامهريزى بيان ثن

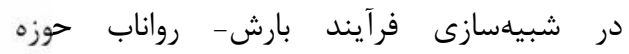

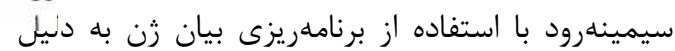

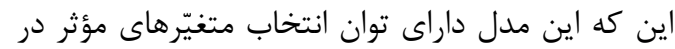

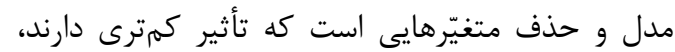

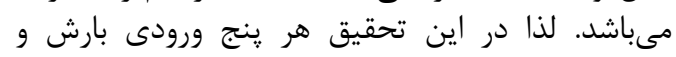

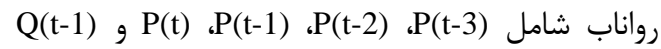
براى تعيين متغيرهاى معنى دار استفاده و براى بران برسى

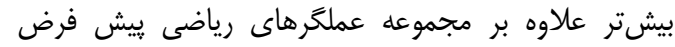

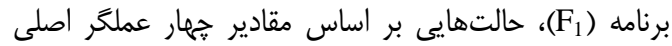

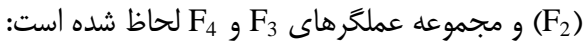

\begin{tabular}{|c|c|c|c|}
\hline \multicolumn{2}{|r|}{ عملكرهاى رنتيكى } & \multicolumn{2}{|c|}{ تنظيمات كلى } \\
\hline.$/ \cdot F Y$ & نرخ جهش & $r$. & تعداد كروموزومها \\
\hline$\cdot 11$ & نرخ وارون سازى & $\wedge$ & اندازه راس \\
\hline$\cdot / 1$ & نرخ ترانهش درج متوالى & r & تعداد زنها در هر كروموزوم \\
\hline$\cdot / 1$ & نرخ ترانهش ريشه درج متوالى & $1 \cdots$ & تعداد جمعيت توليدى \\
\hline$\cdot / r$ & نرخ تركيب تك نقطه ايى & جمع (+) & تابع پييوند \\
\hline$\cdot / r$ & نرخ تركيب دو نقطه ايى & & ت تابع برازش \\
\hline$\cdot 11$ & نرخ تركيب زن & RMSE & معيار خطا \\
\hline$\cdot 11$ & نرخ ترانهش زن & & \\
\hline
\end{tabular}

رواناب با استفاده از مدل برنامهريزى بيان زن را رانشان

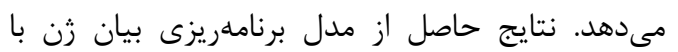

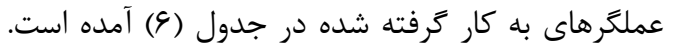

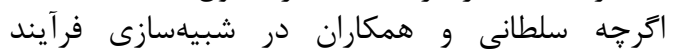
بارش- رواناب روزانه ليقوان، استفاده از مجموعه عملكر فيدر

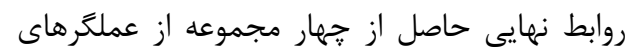

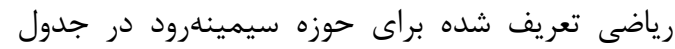

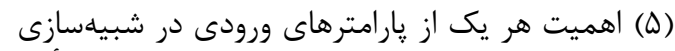

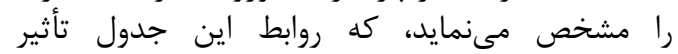

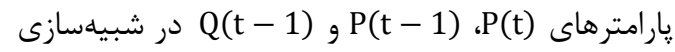


داشتن ساختار ساده رياضى و سهولت محاسبه، با

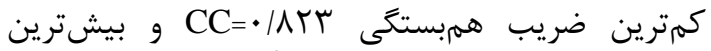
ريشه ميانكين مربعات خطا تملكرد ضعيفترى داشته است. بنابراين در

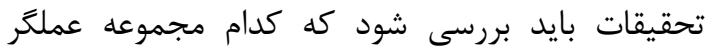

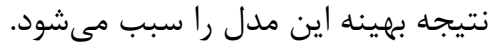

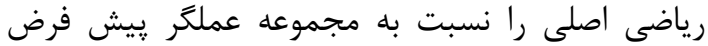

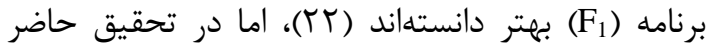

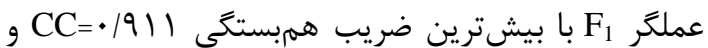

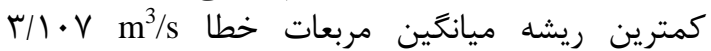
RMSE=

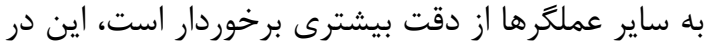

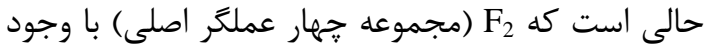

\begin{tabular}{|c|c|}
\hline 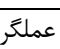 & GEP معادلات \\
\hline $\mathrm{F}_{1}$ & $\left.\mathrm{Q}(\mathrm{t})=\operatorname{Atan}\left[\left(\mathrm{Q}(\mathrm{t}-1)-\mathrm{P}(\mathrm{t})^{6}\right) * \sin (-2.46)^{2}\right)\right]+\sqrt{\sqrt{\mathrm{P}(\mathrm{t})}} * \mathrm{Q}(\mathrm{t}-1)+\sqrt{\mathrm{P}(\mathrm{t})}$ \\
\hline $\mathrm{F}_{2}$ & $\mathrm{Q}(\mathrm{t})=0.29 \mathrm{Q}(\mathrm{t}-1)+0.047 \mathrm{P}(\mathrm{t}-1)+0.11 \mathrm{P}(\mathrm{t})+1.82$ \\
\hline $\mathrm{F}_{3}$ & $\mathrm{Q}(\mathrm{t})=\mathrm{Q}(\mathrm{t}-1)+11.29 \mathrm{Q}(\mathrm{t}-1)-0.96 \mathrm{P}(\mathrm{t})$ \\
\hline $\mathrm{F}_{4}$ & $\mathrm{Q}(\mathrm{t})=-0.79 \mathrm{Q}(\mathrm{t}-1)+0.12 \mathrm{P}(\mathrm{t})+0.8$ \\
\hline
\end{tabular}

\begin{tabular}{|c|c|c|c|c|c|c|}
\hline \multicolumn{3}{|c|}{ صحت سنجى } & \multicolumn{3}{|c|}{ آموزش } & \multirow{2}{*}{ عملكر } \\
\hline $\mathrm{CC}$ & $\begin{array}{l}\text { RMSE } \\
\left(\mathrm{m}^{3} / \mathrm{s}\right)\end{array}$ & NS & $\mathrm{CC}$ & $\begin{array}{l}\mathrm{RMSE} \\
\left(\mathrm{m}^{3} / \mathrm{s}\right)\end{array}$ & NS & \\
\hline .1911 & $r / l \cdot V$ & - MAT & - MFF & g/THE & $\cdot / \Delta \& q$ & $\mathrm{~F}_{1}$ \\
\hline • ATK & F/Tr. & $\cdot|g V|$ & $\cdot / V r V$ & $g / 4 \cdot r$ & $\cdot \mid \Delta F$. & $\mathrm{F}_{2}$ \\
\hline •|AM| & $f / 1 / f$ & $\cdot 19 \wedge 9$ & - /VYq & $G / 4 \Delta V$ & $\cdot|\Delta r|$ & $\mathrm{F}_{3}$ \\
\hline - IAFF & $r / q 4$. & • MIT & •/VTI & $G / 4 \Delta V$ & $\cdot|\Delta r|$ & $\mathrm{F}_{4}$ \\
\hline
\end{tabular}

فرآيند يادگيرى شبكه ... إ در نظر گرفته شده و

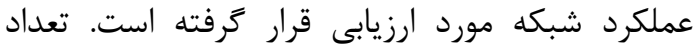

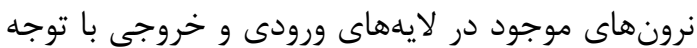

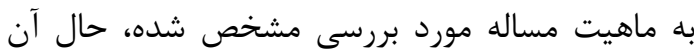

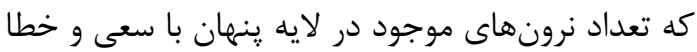

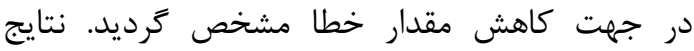
حاصل از فرآيند مدل سازى در جدول (V) ارائه شده

\section{شبكههاى عصبى مصنوعى}

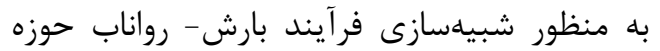

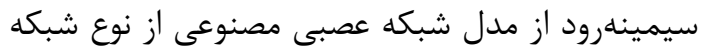

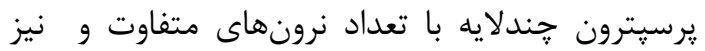

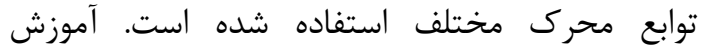

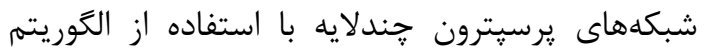

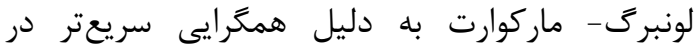
آموزش شبكه استفاده كرديد. تعداد تكرارهاى لازم در

جدول V- ساختار و توابع محرك بهينه شبكههاى عصبى و شاخصهاى آمارى در مراحل آموزش و صحتسنجى

\begin{tabular}{|c|c|c|c|c|c|c|c|c|}
\hline \multicolumn{2}{|l|}{ تست } & \multicolumn{3}{|c|}{ آموزش } & \multicolumn{2}{|c|}{ تابع محرك } & \multirow[b]{2}{*}{ ساختار } & \multirow[b]{2}{*}{ شماره } \\
\hline $\begin{array}{l}\text { RMSE } \\
\left(\mathrm{m}^{3} / \mathrm{s}\right)\end{array}$ & NS & $\mathrm{CC}$ & $\begin{array}{l}\text { RMSE } \\
\left(\mathrm{m}^{3} / \mathrm{s}\right)\end{array}$ & NS & لايه خروجى & لايه (هاى) ينهان & & \\
\hline $4 / 1 \cdot 9$ & $.19 \vee \wedge$ & . /VF4 & GITAT & $\cdot \mid \Delta \Delta \phi$ & سيخموئيد & سيخموئيد & $\Delta-Y-1$ & 1 \\
\hline$r / \Delta 9 \Lambda$ & ./VG. & $\cdot / / 94$ & $\Delta / 991$ & . & سيخموئيد & تانزانت هييربوليك & $\Delta-Y-1$ & t \\
\hline$\varphi / \cdot \Delta \Delta$ & .1990 & - /VAr & g/r.. & $\cdot 1091$ & سيخموئيد & سيخموئيد & $\Delta-\Delta-1$ & r \\
\hline$r / 9 \cdot 1$ & $\cdot / V \Delta Q$ & $\cdot / \lambda \cdot V$ & $\Delta / \Delta \& \Lambda$ &.$|9 \Delta|$ & سيكموئيد & تانزانت هييربوليك & $\Delta-\Delta-1$ & f \\
\hline$F / T T_{D}$ & $\cdot 19 \wedge \Delta$ & - /VFe & G/TVV & $\cdot / \Delta Q V$ & سيخموئيد & سيكَموئيد & $\Delta-V-1$ & $\Delta$ \\
\hline 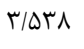 & $\cdot / V 9 \Lambda$ & $\cdot / \Lambda \cdots$ & D/GGY & .1949 & سيخموئيد & تانزانت هييربوليك & $\Delta-V-1$ & द \\
\hline F/TVT & .1994 & - IVAT & G/T. & .1090 & سيخموئيد & تانزانت هييربوليك & $\Delta-r-r-1$ & $v$ \\
\hline r/GAV & $\cdot / V F A$ & $\cdot 11 \cdot 9$ & $\Delta / \Delta \Gamma \Lambda$ & $.19 \Delta \Delta$ & سيخموئيد & تانزانت هييربوليك & $\Delta-\Delta-\Delta-1$ & $\wedge$ \\
\hline$r / \Delta T \Delta$ & $\cdot / V V$ & $\cdot / A \cdot F^{f}$ & $\Delta / G \cdot \Delta$ &. $\mid q 4 V$ & سيخموئيد & تانزانت هييربوليك & $\Delta-V-V-1$ & 9 \\
\hline$r / r \Delta q$ & $\cdot / v 91$ & $\cdot \mid 1 \cdot 1$ & $\Delta / 9 \Delta T$ &.$|94|$ & سيخموئيد & تانزانت هييربوليك & $\Delta-1 \cdot-1 \cdot-1$ & 1 . \\
\hline$r / 9 \Delta 9$ & - IVAT & $\cdot 11 \cdot 9$ & $\Delta / \Delta r q$ & $.19 \Delta \Delta$ & سيخموئيد & تانزانت هييربوليك & $\Delta-11-11-1$ & 11 \\
\hline
\end{tabular}


$1 \cdot$ مقايسه عملكرد ماشين بردار يشتيبان با ساير مدلهاى هوشمند در شبيهسازى فر آيند بارش - رواناب .

مقايسه عملكرد مدلها

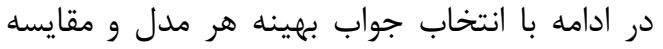

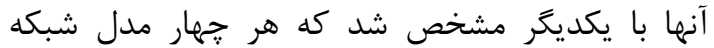

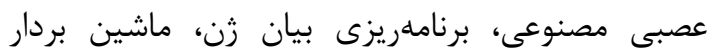

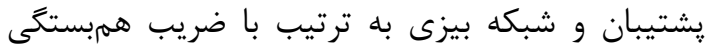

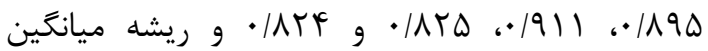

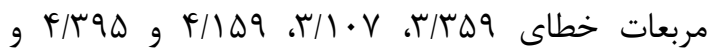

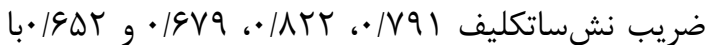
دقت قابل قبولى فر آيند بارش - رواناب حوزه سيمينهاندرود

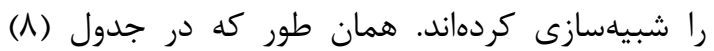

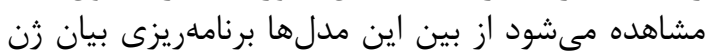

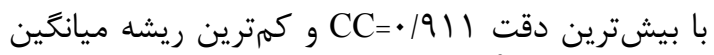

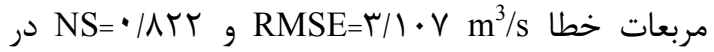
مرحله صحتسنجى در مقايسه با ساير مدلها دارئا بهترين

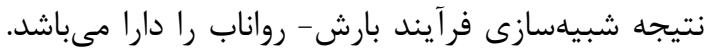

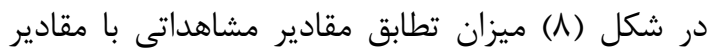

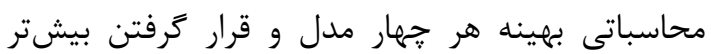

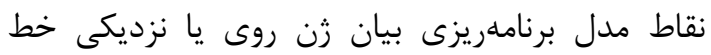

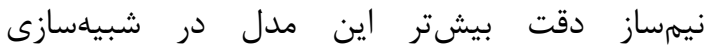
بارش - رواناب را نشان مى دهد. بيثن
ابتدا با در نظر گرفتن سه لايه و تغيير تابع محرك

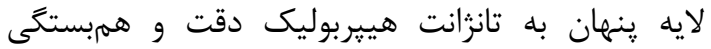

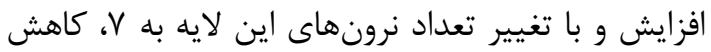

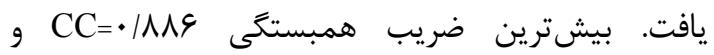

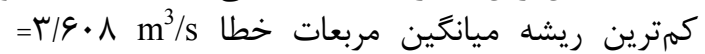
RMSE

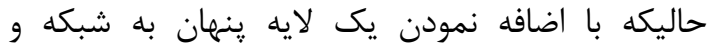

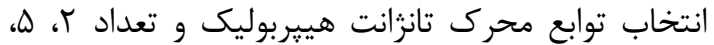

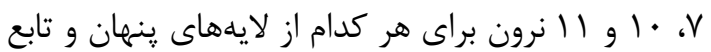
محرى سيخموئيد براى لايه خرونى هرئ، ساختار شماره

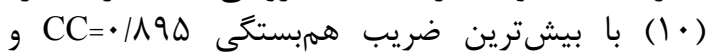

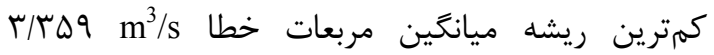

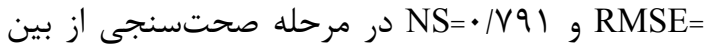
تمام ساختارهاى سه و جههار لايه عملكرد بهترى از خرح نشان داد. در تحقيق انجام شده از سوى صفشكن واد و همكاران

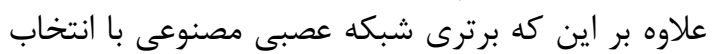

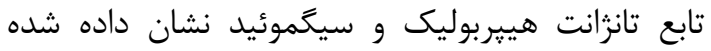

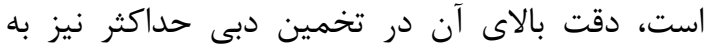

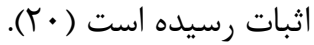

\begin{tabular}{|c|c|c|c|c|c|c|}
\hline \multicolumn{3}{|c|}{ صحت سنجى } & \multicolumn{3}{|c|}{ آموزش } & \multirow[b]{2}{*}{ مدل } \\
\hline $\mathrm{CC}$ & $\operatorname{RMSE}\left(\mathrm{m}^{3} / \mathrm{s}\right)$ & NS & $\mathrm{CC}$ & $\begin{array}{l}\text { RMSE } \\
\left(\mathrm{m}^{3} / \mathrm{s}\right)\end{array}$ & NS & \\
\hline - IATD & $\varphi / 1 \Delta q$ & $.19 \vee 9$ & $\cdot\left|V^{*}\right|$ & $g / T \Delta F$ & $\cdot|\Delta| Q \&$ & SVM \\
\hline - IATF & $4 / \pi 9 \Delta$ & - |qDT & - MET & G/FTV & - IDFT & BN \\
\hline$\cdot / 911$ & $r / 1 . \varphi$ & - IATt & - NGFF & GITYG & - $/ \Delta F q$ & GEP \\
\hline$\cdot 1190$ & $r / r \Delta q$ & - /v91 & $\cdot \mid \lambda \cdot 1$ & $\Delta / 9 \Delta T$ &.$|84|$ & ANN \\
\hline
\end{tabular}
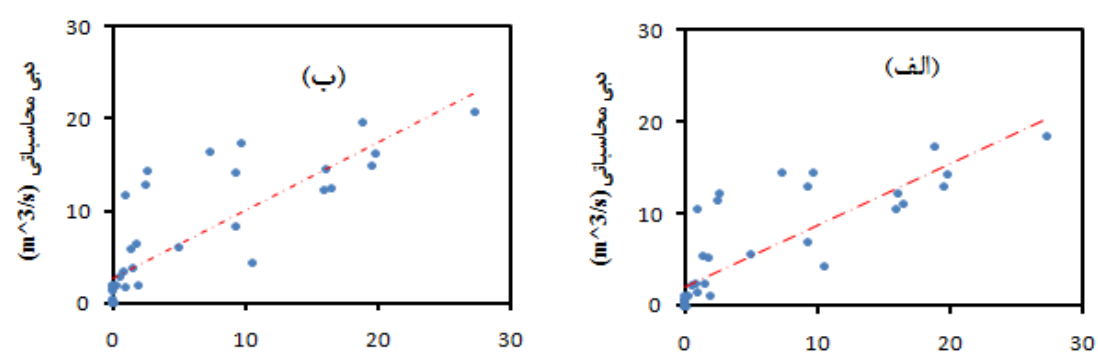

(ابع مشاهداتى (m/s)
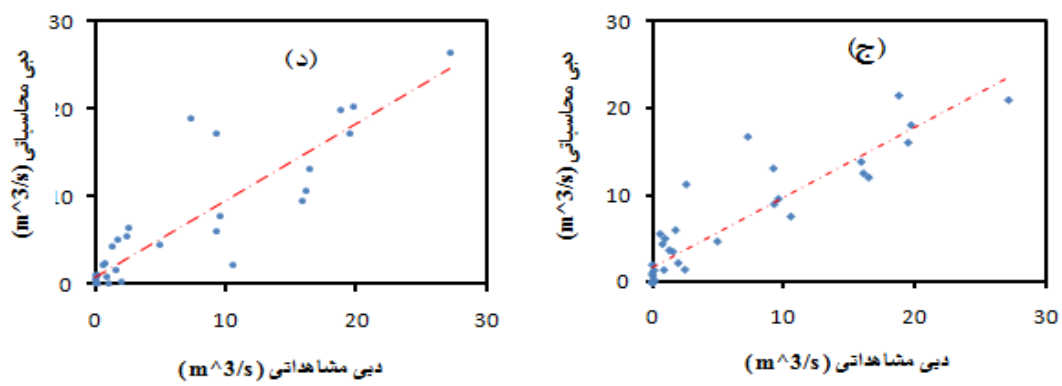

شكل ^- نمودار يراكنش مقادير مشاهداتى - محاسباتى مرحله صحتسنجى: الف) ماشين بردار يشتيبان، ب) شبكه بيزى، ج)

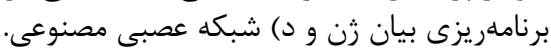


مدلسازى مقادير حداكثر عملكرد خوبى باد همراه

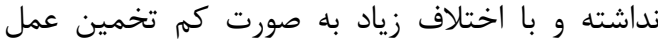

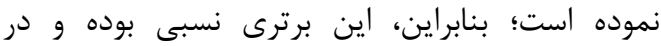

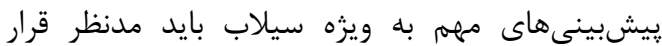

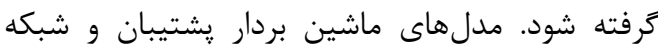

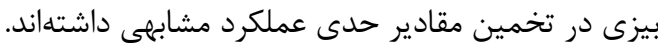

شكل 9 نيز نشان مىدهد مدل بهينه جهار لايه

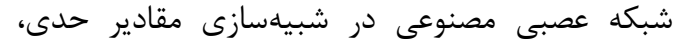
بويزه حداكثر نسبت به هر تجهار مدل بهيتر عمل عمل كرده

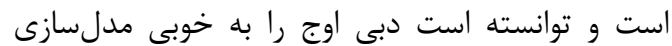

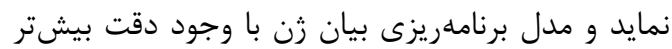

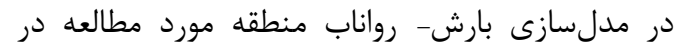

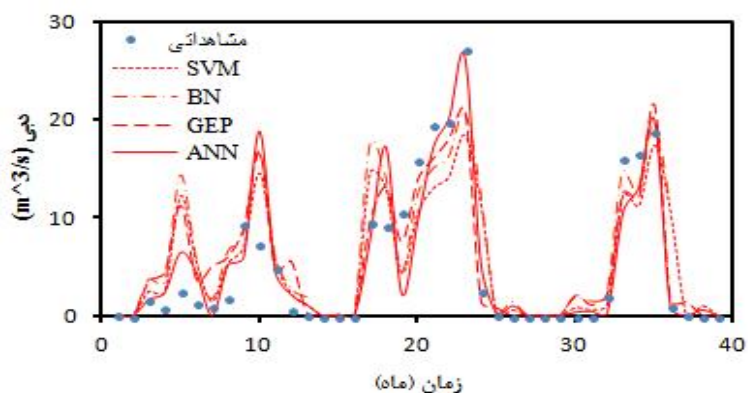

شكل 9- نمودار رواناب مشاهداتى و محاسباتى هجار مدل ماشين بردار پشتيبان، شبكه بيزى، برنامهريزى بيان زن و شبكه عصبى مصنوعى.

نتايج به دست آمده با تحقيق آيتك و همكاران (1) نان

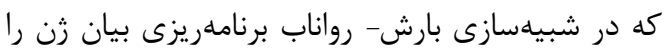

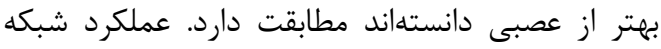

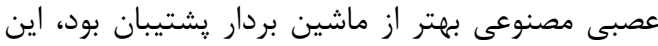

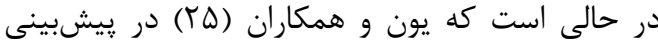

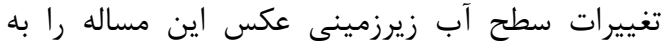

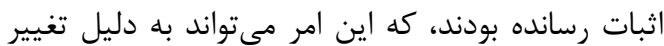

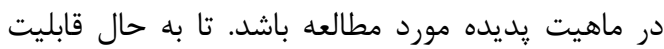

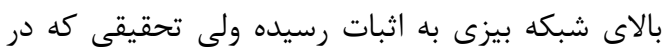

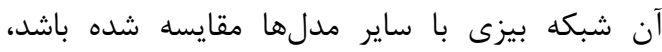

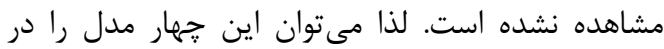

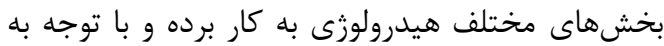

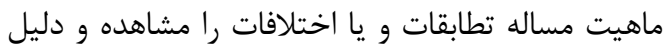
آن آن راهيت مورد بررسى قرار دابقات

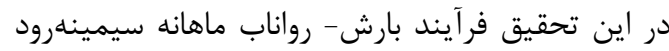

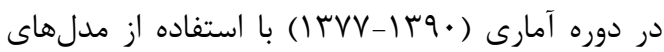

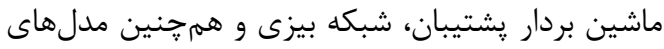

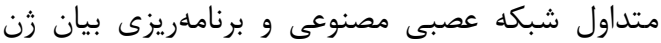

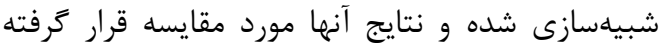

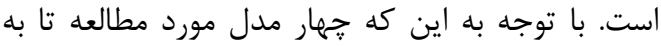

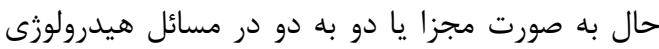

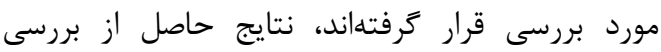

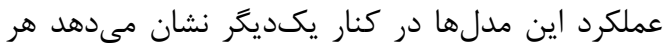

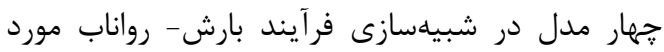

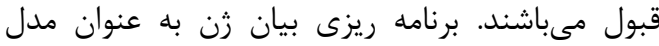

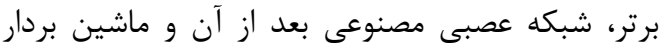

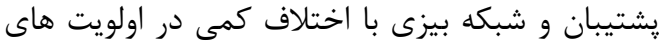
بعدى قرار گرفتند. Aytek, A., M. Asce and M. Alp. 2008. An Application of Artificial Intelligence for Rainfall-Runoff Modeling. Journal of Earth System Science. 117: 145-155.

2. Botsis, D., P. Latinopoulos and K. Diamantaras. 2011. Rainfall-Runoff Moeling Using Suport Vector Regression and Artificial Neural Networks. 12th International Conference on Environmental Science and Technology (CEST2011), Rhodes, Greece, 8-10 September.

3. Carmona, G.J., L. Molina, J. Bromley, C. Varela-Ortega and J.L. Garcia-Arostegu. 2011. ObjectOriented Bayesian Network for Participatory Water Management: Two Case Studise in Spain Jornal of Water Resources Planning and Management. 137: 366-376.

4. Dibike, Y.B., S. Velikov, D. Solomatine and M.B. Abbort. 2001. Model Induction with of Support Vector Machines: Introduction and Applications Journal of Computing in Civil Engineering. 15: 208216.

5. Eskandari, A., R. Noori, H. Meeraji and A. Kiaghaderi. 2011. Development of an Appropriate Model Based on Artificial Neural Network and Support Vector Machine to predict the 5-Day Biochemical Oxygen Demand while. Ecology. 38: 71-82.

6. Farbodfam, N., M.A. Ghorbani and M.T. Aalami. 2010. Stream Flow Forecasting Using Genetic Programming Planning .Journal of Agricultural Knowledge. 19: 107-123.

7. Farmani, R., H.J. Henriksen and D. Savic. 2009. An Evolutionary Bayesian Belif Network Methodology for Optimum Management of Groundwater Contamination. Environmental. Modelling \& Software, 24: 303-310. 
8. Ghorbani, M.A., R. Khatibi, H. Asadi and P. Yousefi. 2012. Inter-Comparison of an Evolutionary Programming Model of Suspended Sediment Time-Series with Other Local Models. In: V. Sebastian (Ed.), Genetic Programming-New Approaches and Successful Applications, In Tech, DOI: 10.5772/47801. 255-284.

9. Ghorbani, M.A., V.P. Singh, R. Daneshfaraz and M. Hasanpour Kashani. 2012. Modelling Pan Evaporation Using Genetic Programming. Journal of Statistics: Advances in Theory and Application, 8: $15-36$.

10. Jayawardena, A.W., N. Muttil and T.M.K.G. Fernando. 2005. Rainfall-Runoff Modeling Using Genetic Programming. International Congress on Modeling and Simulation Society of Australia and New Zealand December 2005, 1841-1847.

11. Khatibi, R., L. Naghipour, M.A. Ghorbani, M.S. Smith, V. Karimi, R. Farhoudi, H. Delafruz and H. Arvanaghi. 2012. Developing a Predictive Tropospheric Ozone Model for Tabriz. Atmospheric Environment, 68: 286-294.

12. Khatibi, R., L. Naghipour, M.A. Ghorbani and M.T. Aalami. 2012. Predictability of Relative Humidity by Two Artificial Intelligence Techniques Using Noisy Data from Two Californian Gauging Stations. Neural Computing and Application, 13: 643-941.

13. Khatibi, R., M.A. Ghorbani, M.T. Aalami, K. Kasim, O. Makarynskyy, D. Makarynskyy and M. Alinezhad. 2011. Ocean Dynamic, 61: 1797-1807.

14. Koza, J.R. 1992. Genetic Programming: On the Programming of Computers by Means of Natural Selection. Cambridge. MIT Press. ISBN 0-262-11170-5, 813 pp.

15. Mediero, L.L. Garrote and F. Martin-Carrasco. 2007. A Probabilistic Model to Support Reservoir Operation Decisions during Flash Floods. Hydrological Sciences Journal, 52: 523-537.

16. Misra, D.T. Oommen, A. Agarwal and S.K. Mishra. 2009. Application and Analysis of Support Vector Machine Based Simulation for Runoff and Sediment Yield Bios stems Engineering. 103: 527535.

17. Mohajerani, H. and M. Kholghi. 2013. Aquifer Based on Bayesian Network is a Management Decision. Journal of Water and Soil, 26: 1522-1534.

18. Noori, R., A. Khakpoor, M. Dehghani and A. Farrokhnia. 2012. Monthly Flow Forecasting Using Support Vector Machine Based on Principal Component Analysis. Water and Waste, 1: 118-123.

19. Sadeghi Hesar, A., H. Tabatabaee and M. Jalali. 2012. Monthly Rainfall Forecasting Using Bayesian Belif Network. International Research Journal of Applied and Basic Sciences, 3: 2226-2231.

20. Safshekan, F., N. Pirmoradian and R. Sharifian. 2012. Hydrograph Simulated Rainfall-Runoff Due to Rainfall Patterns and the Use of Artificial Neural Networks in the Catchment as Kasilian Watershed Management Science \& Engineering, 5: 1-10.

21. Shahbazi, A., B. Zahraei and M. Naseri. 2012. Seasonal Meteorological Drought Prediction Using Support Vector Machine. Water and Waste, 2: 73-85.

22. Soltani, A., M.A. Ghorbani, A. Fakherifard, S. Darbandi and D. Farsadi. 2010. Genetic Programming and Its Application in Rainfall-Runoff Modeling. Journal of Water and Soil Knowledge, 20: 61-71.

23. Taheri, H. and M. Ghafouri. 2012. Comparison between Active Learning Method and Support Vector Machine for Runoff Modeling. Journal of Hydrology and Hydromechanics. 1: 16-32.

24. Tokar, A.S. and P.A. Johnson. 1999. Rainfall- Runoff Modeling Using Artificial Neural Networks. Journal of Hydrology Engineering, 3: 232-239.

25. Yoon, H., S.C. Jun, Y. Hyun, G.O. Bae and K.K. Lee. 2011. A Comparative Study of Artificial Neural Networks and Support Vector Machines for Predicting Groundwater Levels in a Coasta Laquifer Journal of Hydrology, 396: 128-138. 


\title{
Comparison of the Performance of Support Vector Machine with other Intelligent Techniques to Simulate Rainfall-Runoff Process
}

\author{
Mohammad Ali Ghorbani ${ }^{1}$, Atefe Azani ${ }^{2}$ and Leyla Naghipour ${ }^{3}$ \\ 1- Associate Professor, Tabriz University (Corresponding author: ghorbani@ tabrizu.ac.ir) \\ 2 and 3- M.Sc. Student and M.Sc., Tabriz University \\ Received: August 3, $2013 \quad$ Accepted: December 11, 2013
}

\begin{abstract}
Simulation of rainfall-runoff process is a major step in water engineering studies and water resources management. In this study, the rainfall-runoff process of the Siminehroud monthly (1377-1390) were simulated using Support Vector Machines (SVM) with Radial Basis kernel Function, Polynomial and linear Bayesian Network (BN) with a PC Learning Algorithm, also conventional methods such as Artificial Neural Networks (ANNs) and Gene Expression Programming (GEP) were used; finally, the results were compared with each other. Correlation Coefficient (CC), Root Mean Square Error (RMSE) and Nash-Sutcliff coefficient (NS) were used to evaluate the performance of the models. The results indicate the acceptable performance of the models and GEP model shows the highest $\mathrm{CC}(\mathrm{CC}=0.91)$, minimum RMSE $(\mathrm{RMSE}=$ $1.3 \mathrm{~m}^{3} / \mathrm{s}$ ) and $\mathrm{NS}=0.82$ in verification stage.
\end{abstract}

Keywords: Artificial Neural Networks, Bayesian Networks, Gene Expression Programming, Rainfall-Runoff, Siminehroud, Support Vector Machine 